\title{
Responding to the Psychological Needs of Health-Care Workers During the COVID-19 Pandemic: Case Study from the Medical College of Wisconsin
}

\author{
Kristin H. Kroll ${ }^{1,7}$. Sadie Larsen ${ }^{2,3} \cdot$ Kelsey Lamb ${ }^{4}$. W. Hobart Davies ${ }^{5}$. David Cipriano ${ }^{2}$. Terri A. deRoon-Cassini ${ }^{6}$. \\ Himanshu Agrawal ${ }^{2}$. Deepa Pawar ${ }^{2} \cdot$ Julie Owen $^{2} \cdot$ Jennifer N. Apps ${ }^{2}$
}

Accepted: 22 May 2021 / Published online: 31 May 2021

๑) The Author(s), under exclusive licence to Springer Science+Business Media, LLC, part of Springer Nature 2021

\begin{abstract}
With the advent of the novel coronavirus (COVID-19) pandemic, health-care workers have been faced with an inordinately high level of trauma as frontline providers. The Medical College of Wisconsin (MCW) partnered with affiliate hospitals and community partners to mobilize a matrix of available support and interventions to deliver psychological services to reach all levels of health-care providers in timely, accessible formats. While virtual peer support groups were the most utilized resource among the support group options, other opportunities also provided unique benefits to learners whose education had been disrupted by the pandemic. Mental health must be prioritized for health-care workers in the event of future public health crises. Lessons learned from this pandemic indicate that it is critical to involve learners early on in the process in order to meet their educational needs and to increase access to evidence-based care.
\end{abstract}

Keywords Coronavirus $\cdot$ Pandemic $\cdot$ Health-care workers $\cdot$ Training

\section{Introduction}

Health-care workers (HCWs) are a critical part of the response to all phases of disasters, and they face exposure to both traumatic and non-traumatic stressors in this role (Brooks et al., 2019; Center for the Study of Traumatic Stress, 2020; Surya et al., 2017). The literature from past

Kristin H. Kroll

khkroll@mcw.edu

1 Department of Pediatrics, Medical College of Wisconsin, Milwaukee, WI, USA

2 Department of Psychiatry and Behavioral Medicine, Medical College of Wisconsin, Milwaukee, WI, USA

3 Milwaukee VA Medical Center, Milwaukee, WI, USA

4 School of Medicine, Medical College of Wisconsin, Milwaukee, WI, USA

5 Department of Psychology, University of Wisconsin-Milwaukee, Milwaukee, WI, USA

6 Division of Trauma and Acute Care Surgery, Department of Surgery, Medical College of Wisconsin, Milwaukee, WI, USA

7 Medical College of Wisconsin, Wauwatosa, WI, USA disasters and the current COVID-19 pandemic demonstrates that HCWs experience psychological effects related to these stressors (Lai et al., 2020; Lee et al., 2018; Maunder et al., 2003; Paladino et al., 2017). For instance, a recent metaanalysis found that about $20 \%$ of HCWs, compared to $10 \%$ of the general population, had post-traumatic stress symptoms or post-traumatic stress disorder during or after a coronavirus outbreak (Salehi et al., 2021).

Further studies identified the importance of psychological interventions in helping HCWs to handle both the acute and long-term distress associated with viral respiratory disease outbreaks, such as the 2003 SARS global outbreak (Epstein \& Krasner, 2013; Tawfik et al., 2018). A brief review of the disaster mental health literature reveals a myriad of interventions designed to support disaster survivors, some of which have also been utilized to support HCWs and first responders. SAMHSA (2015) published a Disaster Behavioral Health Interventions Inventory, which highlighted evidenceinformed or evidence-based early interventions. Some of the interventions included in this inventory were acute phase interventions such as Psychological First Aid (PFA), intermediate phase interventions such as Cognitive Behavioral Therapy (CBT) and Skills for Psychological Recovery, and 
long-term interventions such as CBT and Eye Movement Desensitization and Reprocessing (EMDR).

Brooks et al. (2019) also cited evidence to support the use of PFA, CBT, and peer support interventions for traumaexposed employees such as HCWs. Similarly, Surya et al. (2017) underscored interventions for HCWs such as PFA, team-building, mindfulness, relaxation response training, physical exercise, as well as other techniques based on principles of CBT. In addition to specific interventions, some of the disaster literature identified the critical role of managers in supporting their employees during trauma or disaster to enhance individual and organizational resilience (Brooks et al., 2019; Louise Duncan, 2020).

\section{The Coronavirus (COVID-19) Pandemic}

A new coronavirus was identified in Wuhan, China in December 2019, and this new virus (SARS-CoV-2) quickly spread across the world (Wu et al., 2020). In January 2020, the first case of COVID-19 was identified in the United States, with community spread to all 50 states occurring within the next 2 months (Omer et al., 2020). Following the onset of this pandemic, a rapidly growing body of evidence began to focus upon the psychosocial impact of COVID-19 on HCWs and learners.

COVID-19 posed unique challenges to HCWs, including the rapid spread of infection, severity of patient illness, high patient volumes, limited resources and supplies, rapidly changing best-practice guidelines, and hospital staffing shortages related to infection rates of and quarantine requirements for HCWs (Tracy et al., 2020). The psychological impact of COVID-19 upon HCWs was variable, for instance, among HCWs, being a nurse and being female appeared to confer greater risk. However, these studies indicated that HCWs were at greater risk for anxiety, depression, and traumatic stress-related disorders, when compared to the general population (Cabarkapa et al., 2020).

The pandemic also quickly revealed increased challenges for medical students. A recent survey of over 7100 Changzhi Medical College students in Shanxi, China, demonstrated that nearly $25 \%$ of students reported anxiety symptoms related to the COVID-19 outbreak (Cao et al., 2020). Thus, interventions for HCWs must provide a multifaceted strategy, including psychological services to prevent and mitigate the psychological impact of the COVID-19 pandemic on HCWs and medical learners to ensure individual and health system resilience and capacity, both now and in the future (IASC, 2020; Institute of Medicine, 2012; Surya et al., 2017; WHO, 2020).

In Wuhan, the stressors and symptoms experienced by ICU nurses were addressed via the provision of specialized training and communication efforts, adjustments in work shifts to allow for adequate rest periods, early psychological assessments and interventions, and the establishment of social care and support groups (Shen et al., 2020). Representatives in the National Health Service (NHS) specified three key strategies to protect the mental health of HCWs on the front lines of the COVID-19 pandemic, including enhanced decision-making support to mitigate complex decisions that could lead to moral injury, increased time provided to HCWs both to rest and to process distressing events, and consistency in staffing to allow for the development of supportive, trusting relationships and to facilitate easier recognition of distress among colleagues (Roycroft et al., 2020).

A tiered approach grounded in a public health prevention model has been proposed by Tracy et al. (2020). This model encourages three levels of prevention, including primary, secondary, and tertiary, to meet HCWs at their level of distress. The first tier suggests encouraging the provision of clear information, briefings, and reflection as well as an optimization of the work environment as primary prevention strategies. Secondary prevention involves early identification of distress and peer support protocols. Active monitoring with rapidly and easily accessible mental health services are recommended as a tertiary prevention strategy.

\section{COVID-19 and the Medical College of Wisconsin Academic Community}

In March 2020, the Medical College of Wisconsin (MCW) saw a sudden upheaval in its ability to deliver clinical and educational services. As the COVID-19 pandemic spread, radical decisions were made across the enterprise for the sake of patient and employee safety, wherein all ambulatory centers ceased in-person visits. Simultaneously, medical students were withdrawn from rotation sites across the enterprise, and in its place, a virtual curriculum was developed across all medical specialties. Statewide "Safer at Home" orders were carried out, which added to the magnitude of this change for consumers and providers alike.

Health-care learners found themselves in a unique position among their peers and mentors during the pandemic. They were in training to be frontline personnel but were not yet fully vested in that role. As frontline providers in the hospital, resident physicians were placed in the role of "hero" nearly overnight, without enlisting in such a job, or for that matter, receiving the pay of a fully licensed physician. This resulted in great heterogeneity in responses to the COVID19 crisis among health-care learners.

The pandemic caused some medical students to question their choice of profession. Some learners eloquently described their original motives for entering medical school being tied to a desire to help and care for others, while at the same time feeling torn about making the sacrifice of their own health or possibly infecting loved ones. Anecdotally, 
some students expressed relief that the impact of the coronavirus was minimal due to the particular point they were at in their training. On the other hand, students and residents alike expressed guilt at seeing their classmates struggle or for not being out on the front lines. Many advocated for more of an active role in the health-care response, while their education was on pause.

Many health-care learners were concerned about being 'sidelined' from direct patient contact, as many training programs initially chose to do so to protect the health and safety of learners. Anxiety centered upon missing out on vital clinical experiences that were valuable for training. At MCW, health psychology doctoral interns were still in need of both direct hours of experience, as well as a way to continue developing needed competencies with the onset of the pandemic. Such experience is critical for being a viable candidate for competitive residencies, fellowships or jobs.

Among medical students, the USMLE Step 1 exam, taken by second year students as the first step in their licensing, was significantly disrupted during this pandemic. Due to social distancing requirements, most exams were canceled or postponed, leaving learners with a great deal of uncertainty about how to maintain and plan their studying without being certain of test dates. Other licensure and national exams have posed similar challenges. Interviews for internships and residencies became virtual, leaving learners feeling at a disadvantage.

Given these wide-ranging difficulties and needs, as well as the general mental health vulnerability of learners at baseline (Rotenstein et al., 2016), supporting the learning needs of medical students, residents, and health psychology learners became a priority early in the pandemic. As such, after each learner co-facilitator led a group session, they then met with their faculty co-facilitator in a group setting to help process their own experience of facilitating the group session. Separate support groups were also offered for students and these were facilitated by a psychologist and a psychiatrist. In addition, the psychology interns were able to receive additional support in their weekly supervision.

Like many academic medical centers, concerns about shortages of personal protective equipment (PPE), compounded by financial, psychological and moral stressors from furloughs or other financial mitigations added to the cumulative distress within the MCW community. Acknowledging these concerns and issues helped all parties join together and collaborate in responding to the unprecedented demands that this public health crisis was imposing upon HCWs. It was quickly recognized that individuals across the institution, in all types of roles, would need emotional support.

These institution-specific concerns fit with those identified in the broader literature. For instance, one study of listening sessions conducted with HCWs and learners indicated eight sources of COVID-related anxiety: lack of access to adequate PPE, exposure to COVID-19 and the possibility of infecting loved ones, lack of access to/availability of testing, uncertainty surrounding organizational support, access to childcare in the setting of increased work hours and school closures, ability to perform competently if re-deployed into a new practice setting and/or role, and lack of access to up-todate information and communication (Shanafelt et al., 2020).

From these eight identified sources of COVID-related anxiety, Shanafelt and colleagues created the following themes to summarize the HCWs' and learners' request for help from their respective institutions: "hear me, protect me, prepare me, support me, and care for me." Likewise highlighting the role of leadership at institutions, Greenberg et al. (2020) opined in a recent analysis: "Whether someone develops a psychological injury or experiences psychological growth is likely to be influenced by the way that they are supported before, during, and after a challenging incident."

Finally, in addition to supporting faculty, staff, and learners, a third arm of the support process at MCW involved providing support to community health-care providers, both by decreasing distress and by building resilience. This latter goal was chosen due to the impact of resilience-building in helping to reduce stress and prevent burnout, which has been associated with errors in medical decision-making (Tawfik et al., 2018). These two aims emphasized the dual importance of both primary and secondary prevention when it comes to the well-being of HCWs.

As such, MCW's efforts focused upon primary prevention, also known as the aim to prevent the actual occurrence of a disease or illness such as traumatic stress, as well as secondary prevention, which aims to intervene early when symptoms are present in order to have the most optimal prognosis (Magruder et al., 2016). In one study that investigated the detrimental psychological impact of pandemics and epidemics upon HCWs, the authors recommended the promotion of resilience trainings and the utilization of online support services to reduce the impact upon HCWs (Preti et al., 2020). Therefore, the goal of MCW's different intervention strategies were to provide interventions to address the current concerns and needs of all types of HCWs, while also providing opportunities to build resilience.

\section{Current Study}

With the goal of providing psychological support services to HCWs and learners to optimize resilience in the face of the COVID-19 pandemic, MCW partnered with affiliate hospitals and community partners, including the institution's tertiary care center, ambulatory clinics, children's hospital affiliate, and the Veteran's Affairs Medical Center, to mobilize a matrix of available supports and interventions designed to reach all levels of HCWs (e.g., environmental services 
staff, respiratory therapists, nurses, medical students, resident physicians, physician faculty, etc.) in timely, accessible formats. The methods utilized by MCW can provide a helpful model for addressing local needs following a disaster via the delivery of various virtual psychological services.

\section{Methods}

\section{Sample}

Our sample focuses on three primary groups of participants affected by the pandemic and supported by the institution (See Fig. 1). First, peer support was provided for healthcare workers within the academic community (both at MCW and at affiliated institutions). Second, health-care learners were involved in both providing support to others (as a way to continue to develop competency) and also in receiving support themselves. Third, MCW rallied to support healthcare workers in the community who may not have as much built-in support, yet may be at risk for increased pandemic distress.

\section{Procedure}

\section{Providing Peer Support for HCWs within the Academic Community}

In response to the concern about supporting HCWs, MCW's Department of Psychiatry quickly partnered with Human
Resources and a center within MCW dedicated to physician development and training to develop virtual support groups to provide a resource for coping with stress. Given that the Department of Psychiatry was involved in the creation of the MCW support groups, facilitators were recruited on a voluntary basis from the faculty of licensed psychologists and psychiatrists. Medical students and health psychology residents volunteered to serve as co-facilitators. Every facilitation team consisted of one to two behavioral health specialists (i.e., health psychology resident or faculty member) paired with one medical student. Both facilitators and co-facilitators received an informational handbook reviewing PFA and support group basics, as well as guidelines on conducting peer support groups. In order to meet a wide variety of needs, groups were created based on similarity of health-care job and then identified as meeting on certain days/times.

Briefly, PFA involves mental health providers being available for rapid assessments of the immediate needs of individuals soon after trauma (Vernberg et al., 2008). It is designed as a pragmatic approach to connect individuals experiencing high stress with the resources necessary to mitigate that stress and ultimately prevent non-remitting post trauma sequelae. It also is grounded in the idea that not everyone is in need of services, and that therapeutic services are not necessary acutely for the majority of individuals. Instead, the focus is on utilizing active listening and compassion to help others identify coping strategies and social support that can help buffer against distress, while linking individuals to more formal mental health services if necessary.
Fig. 1 Psychological service delivery matrix

\section{Healthcare Workers}

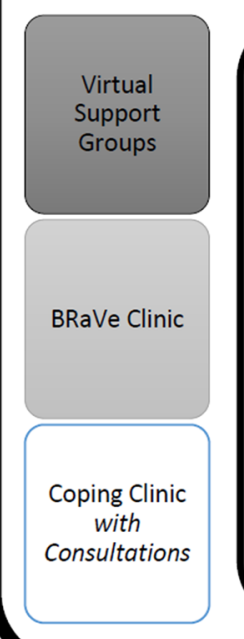

\section{Faculty}

\section{Learners}

BRaVe Clinic

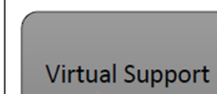

Student

Support

Groups 
By partnering with MCW's Human Resources department, groups were advertised on a broad basis to all faculty and staff, who could then easily request participation through an internet form. The groups were described as "free (independent of the health plan), virtual peer-led support groups for all interested faculty and staff to receive support, coping strategies, share worries, and remember we are not alone during these challenges." The strong response to the initial advertisement necessitated scheduling of additional groups. In addition, when the institution implemented furloughs, additional support groups were added for those specifically impacted by that stressor. In total, 130 people registered to participate in the groups. After each group, group facilitators met with their co-facilitators to review and process the dynamics and content of each group.

Learners within the medical school institution also required additional emotional support. One of the same Psychiatry faculty involved in the larger support group project initiated similar groups that were available to students across all schools of the health sciences university. Facilitators, again, were faculty from the Psychiatry department, and co-facilitators were psychology residents. The format of these sessions was open, without need for advance registration, and the focus of discussion was on maintaining positive emotion and general well-being through principles of positive psychology. Topics of positive psychology included maintaining and enhancing positive emotions through gratitude, forgiveness and optimistic explanatory style (Tugade et al., 2014). Discussion also focused upon sustaining relationships and finding meaning in life and work (Seligman, 2011).

The children's hospital affiliate attempted a different support structure, utilizing drop-in virtual groups that did not require advance registration. Instead, the groups were hosted on a virtual platform that was widely advertised to hospital staff, and anyone interested could log in at two designated times each day, one morning and one evening. Facilitators for these groups included volunteers from the staff psychologists, as well as Child Life specialists, master's prepared therapists, and yoga instructors.

The Veterans Affairs affiliate hospital also created support teams for those HCWs and staff working in the intensive care units or floors dedicated to the care of patients with COVID-19. Groups were designed to provide coping resources as well as emotional support. The facilitators of these groups included psychologists, who also have a dual appointment in the MCW Psychiatry department, as well as psychology interns and fellows.

Additionally, MCW developed a peer support program called "Support our Staff" (SOS), with tiers of support to match levels of distress that occur among HCWs. This program had been implemented prior to the pandemic, in an effort to train identified peers in PFA and provide crisis support in the health-care setting. This existing structure was further adapted during the increased stress of the pandemic to provide check-ins with HCWs on the units caring for patients with COVID-19, in hopes of mitigating emotional burden and identifying specific HCWs that needed increased intervention.

Much like the SOS program described here, a number of more peer-lead programs developed during the pandemic. The Awareness of Resilience in Stressful Events program was designed to offer peer support to staff nurses and appeared to be utilized by nurses experiencing burnout (Connors et al., 2019). A separate video-conferencing group for junior doctors was developed to provide peer support, and which resulted in these participants being able to identify unique needs during the pandemic, such as adequate rest areas, that contributed to well-being during this stressful time (Behrman et al., 2020).

Providing Training Requirements for Health-Care Learners within the Academy Community As described above, our academic community was privileged to have learners volunteer their efforts during the pandemic to both support HCWs and to gain valuable experience in mental health and virtual care. Health-care learners hailing from a multitude of backgrounds and levels of expertise-medical students, psychology interns and postdoctoral fellows alike-were involved in numerous aspects of the response to COVID-19 at MCW. These different types of learners were co-facilitators of the support groups being run for faculty, medical residents and staff. The capacity for virtual intervention, specifically, enabled a great deal of flexibility and opportunity for learners throughout this process.

Medical students were offered the volunteer opportunity to co-facilitate the virtual support groups established for faculty and staff, alongside psychiatric and behavioral health specialists at the institution. The thought process behind this was multifaceted: medical student involvement would serve as an avenue for mental health-related care experience, enable the development of new and valuable skills, and ultimately provide critical connections to medical education community members. Furthermore, it empowered students to find purpose and garner fulfillment in the midst of turbulence. As part of this commitment to involving learners in the virtual provision of care, the "Coping Clinic" was developed. The unique aims of this clinic were to (1) support health-care learners and providers learning to cope with the realities of COVID-19, and (2) provide training opportunities for health psychology learners (practicum students, interns, and postdoctoral fellows) who were initially removed from their inpatient clinical rotations for safety precautions and PPE conservation at the beginning of the pandemic.

Infrastructure was already in place for students to provide ambulatory health psychology services to primary 
care referrals; therefore, this clinic was able to be quickly designed, with services first provided within weeks of learners being physically removed from the health-care system. Additionally, learners were able to gain competency in the new area of telepsychology with training from one of our faculty with prior experience in implementing telepsychology services. Learners were then subsequently able to provide remote psychological health care just as quickly as practicing psychologists.

With this clinic, patients who were HCWs and medical learners received individual telephone or video appointments. Sessions were documented in the medical record similar to any other clinical appointment except without a billing component attached. The focus of individual sessions was on coping with the impact of the COVID-19 crisis and the many ways in which it affected training and life more broadly (e.g., changes to life plans such as weddings, worries about impact on future career, as well as health-anxiety related to the virus itself). The approach was CBT-oriented, skills-focused, and supportive. A rough time limit of 20 sessions was identified, to be continued as long as an individual was still affected by COVID-19-related stressors. Should a referral to longer-term therapy or a higher level of care (e.g., residential) be indicated, providers worked with clients to identify an appropriate community provider. All services were supervised virtually by a licensed psychologist.

Over time, the scope of the clinic was expanded to include frontline providers such as nurses, nursing assistants, respiratory therapists, and environmental service workers, given the understanding that there was an increased mental health burden to both frontline HCWs (Galbraith et al., 2020) and hospital staff. As opposed to traditionally accessed mental health services offered pre-COVID, this clinic was designed to be free to clients in recognition of the health-care system's desire to support its workers and learners and to help them manage their emotional reactions so that they could continue to work effectively in a high stress environment.

Given the recognition that stress levels remained high among HCWs within the hospital, the health psychology program next pivoted to mental health outreach during the end of the second month of the pandemic response, creating the Nurse Manager Consultation Project. This project was collaboratively created with mental health and nursing leadership at the affiliated academic hospital. Nurse Managers met with a psychiatric clinical nurse specialist and were then given information about working with a health psychology resident who served as a mental health consultant to that manager and their team. The resident would meet with the nurse manager to determine what salient stressors and challenges were occurring on the unit and to help the nurse manager effectively improve the mental health of the nursing staff by utilizing resources effectively. This arm of the
Coping Clinic utilized psychology consultative skills to help vulnerable populations cope and access resources as needed.

\section{Providing Care to High-Risk Health-Care Workers in the Community}

Given that the aforementioned clinics were providing highquality care to HCWs within the academic community, an initiative was created to help provide care to health-care colleagues in the larger community that were not affiliated with an academic institution. With this aim, the Building Resilience Virtually (BRaVe) Clinic was developed to help provide individuals, particularly frontline and emergency HCWs, with the opportunity to access evidence-based mental health care via in-home video-conferencing. This clinic was developed to not only address the specific needs of each individual patient, such as depressive or traumatic stress symptoms, but to also help patients build resilience and more effectively manage distress. In order to provide mental health services to individuals in need regardless of insurance status or socioeconomic status, external funding from a local community grant was secured. Patients were eligible to receive care in this virtual clinic if they were assessed as being particularly vulnerable to the negative psychological sequelae of the pandemic, such as individuals at risk of interpersonal violence or with a premorbid history of depression, as well as medical professionals who provide direct medical care to patients diagnosed or being tested for COVID-19 across four counties in a major metropolitan area. Doctoral students from local psychology graduate programs provided clinical care under the supervision of licensed psychologists.

Given that doctoral learners would be providing care, a training protocol was created to ensure that learners were competent to provide trauma-informed telebehavioral health services prior to interacting with any patients. At the start of training, learners were asked to review a recorded webinar on trauma-informed care. Then, they reviewed an hour-long recorded webinar that was created by a licensed psychologist with specialized expertise in telepsychology.

This training was structured to model the interprofessional telebehavioral competencies created by the Coalition for Technology in Behavioral Sciences, which identify the integral knowledge and applicable skills necessary to provide telebehavioral health services (Maheu et al., 2019). This recorded training focused upon clinical factors associated with providing telebehavioral health services to a patient's home, including but not limited to: risks and benefits associated with this service delivery model, legal and regulatory issues in the state of Wisconsin both prior to and following the onset of the COVID-19 pandemic, clinical assessment for appropriateness of fit for a patient to engage in remote psychological services, creation of individualized 
emergency management plans as part of overall risk management planning, clinical documentation pertinent to this service delivery model, ethical and cultural considerations, and strategies for enhancing telepresence.

Following the completion of this training, all doctoral learners had to meet a minimum passing score on a posttraining evaluation in order to ensure they had acquired adequate foundational knowledge about providing ethical and evidence-based telebehavioral health services. To achieve a passing score, learners had to achieve a minimum of $80 \%$ on a ten-item multiple-choice quiz that assessed the content areas above. All five doctoral learners received a passing score on this post-training evaluation on their first attempts. Each doctoral learner was then asked to complete an individual consultation with a telebehavioral expert to demonstrate their logistical skills proficiency in areas such as conducting basic technical trouble-shooting, explaining aspects of informed consent specific to telebehavioral health, and knowledgeably discussing how to create and modify individualized emergency management plans. See Fig. 2 for a visual representation of this training process.

As part of the BRaVe Clinic's commitment to safety, four safety pillars were implemented as part of the design of the clinic: (1) maintain a high ratio of supervision hours to patient contact hours, (2) assign patients to more experienced clinicians if they are at high risk for suicide, (3) provide clinical resources to all learners that provide recommendations on managing high-risk patients remotely and (4) create an individualized emergency management plan with each patient at first contact. Please see Table 1 for more

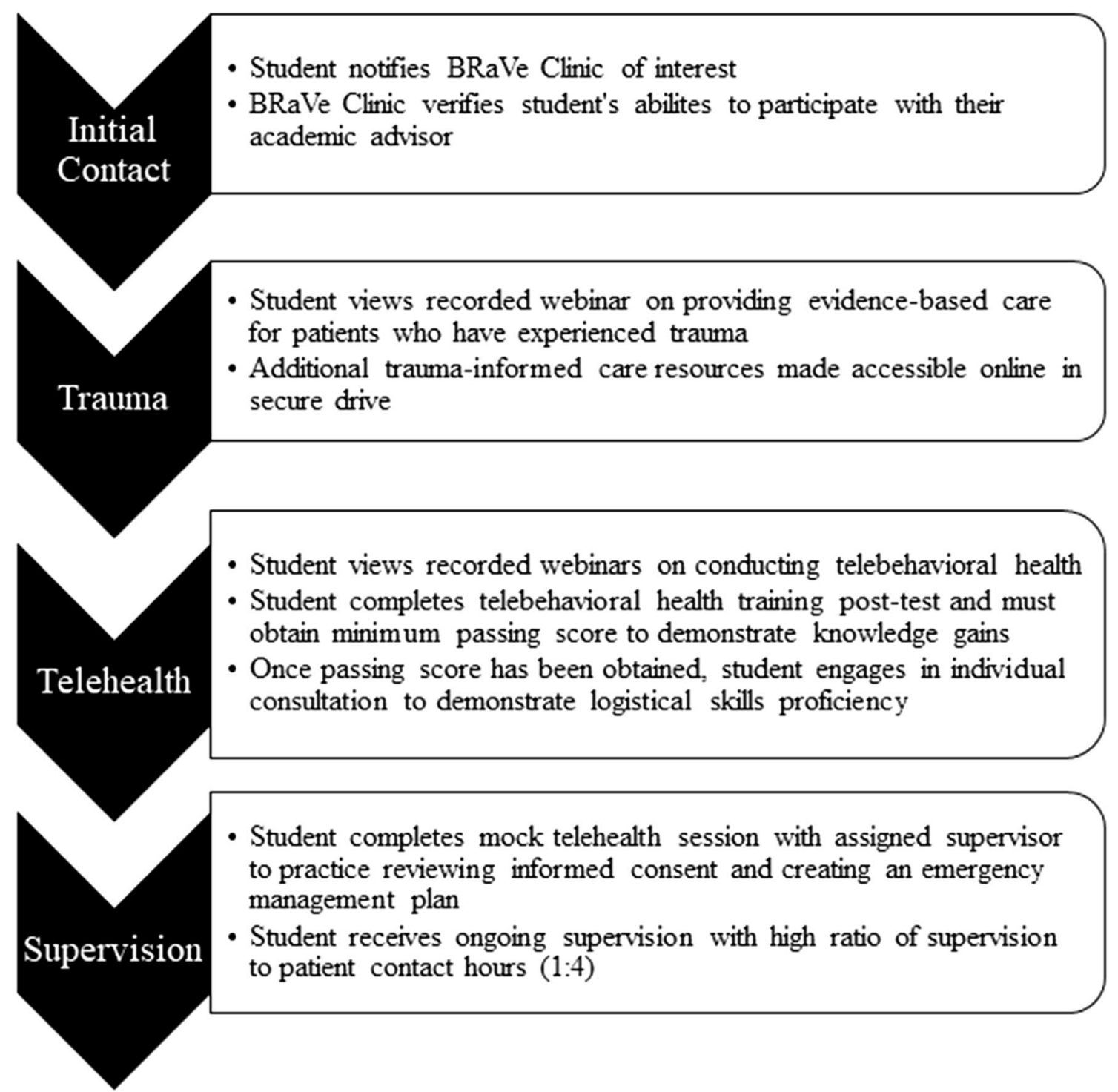

Fig. 2 Student training process for the BRaVe Clinic 
Table 1 Safety management protocol

\begin{tabular}{ll}
\hline Critical task & Description \\
\hline Screening & $\begin{array}{c}\text { Patient assessed for suicidality at initial contact with the Columbia Suicide Severity Rating Scale (Posner } \\
\text { et al., 2011) } \\
\text { Patient identified as being at high risk for suicide was treated by a licensed psychologist rather than a } \\
\text { student trainee }\end{array}$ \\
$\begin{array}{l}\text { An individualized emergency management plan was created with each patient at first initial contact in the } \\
\text { event a psychiatric emergency should arise during the patient's engagement in services }\end{array}$ \\
$\begin{array}{l}\text { High Intensity Supervision } \\
\text { Evidence-Based Clinical Resources }\end{array}$ & $\begin{array}{c}\text { Suicide safety planning resources were made available to all clinicians given evidence of its effectiveness } \\
\text { and appropriateness for telehealth (Brown \& Stanley, 2009; Rojas et al., 2019) }\end{array}$
\end{tabular}

information about the safety management protocol for this clinic.

Outcome data regarding each patient's participation in the BRaVe Clinic was collected, with emphasis upon patient satisfaction, psychiatric symptom reduction, and family stress associated with COVID-19. At the time of this publication, outcome data was continuing to be collected.

\section{Results}

\section{Health-Care Workers}

The virtual peer support groups at MCW were the most utilized resource among support group options, with over 100 individuals registering for groups, more than 40 faculty and learners volunteering to serve as facilitators and supervisors of these groups, and nine groups extending their meeting schedule past the predetermined 8-week duration. This was in contrast to the virtual drop-in sessions held at affiliates, where the number of participants was less than the number of sessions offered, with many sessions having no utilization.

Information was collected on a voluntary basis from those leading the MCW groups regarding facilitator levels of experience and comfort with virtual formats. An overwhelming number of volunteer facilitators reported no prior experience in telemedicine, although despite this the overall comfort level of facilitating the groups was an average of 7.73 on a 1(low)-10(high) scale. Additionally, data assessed overall satisfaction from participants, as well as general themes discussed during group sessions.

While a relatively small number of participants $(n=17)$ completed a post-participation survey, all of those completing the survey reported they would participate in a virtual support group again. The themes reported from participants that were most discussed during the groups included uncertainty, isolation, and change, while the facilitators reported feeling that the most frequent theme of the meetings was coping. Qualitative descriptions were intended to inform on lessons learned from this rapid mobilization of psychosocial support systems implemented across different platforms. Informal feedback and anecdotal feedback suggested that structured, scheduled sessions received the largest participation. Further, the trained facilitator/peer support model was the most utilized resource.

\section{Learners}

MCW leadership recognized the need to creatively serve medical learners during this disruption in their education to continue to promote their professional development, while also having access to care as needed. The student response to the call for volunteers was abundant; medical students at all levels of training with a variety of long-term career goals entered themselves into the role of co-facilitator. Facilitation dynamics varied among teams, but feedback for this model was broadly positive. Behavioral health faculty expressed confidence in their student co-facilitators and value-added from their presence in the peer group. Further, by the conclusion of the initial 8-week block of meetings, medical students were periodically facilitating groups on their own as their skills developed and relationships with group members flourished. Students expressed satisfaction in their growth as medical learners and in their ability to contribute.

Several unique benefits of involving learners in virtual mental health care became evident (See Fig. 3). The format encouraged increased engagement, as learners were able to participate in multiple types of care for multiple communities without being required to be physically present. Specifically, a learner could engage in back-to-back sessions without having to factor in travel or other competing scheduling conflicts. Similarly, a learner could receive increased supervision, as the virtual format permitted multiple supervisors to be present with greater ease. This virtual supervision format also enabled increased support for potentially complicated, high risk, or intimidating patient populations, for instance, providing mental health care to frontline physicians who may be viewed by learners as superiors or authority figures creating a complex patient-provider dynamic. Exposure to this sort of nuanced care is imperative 


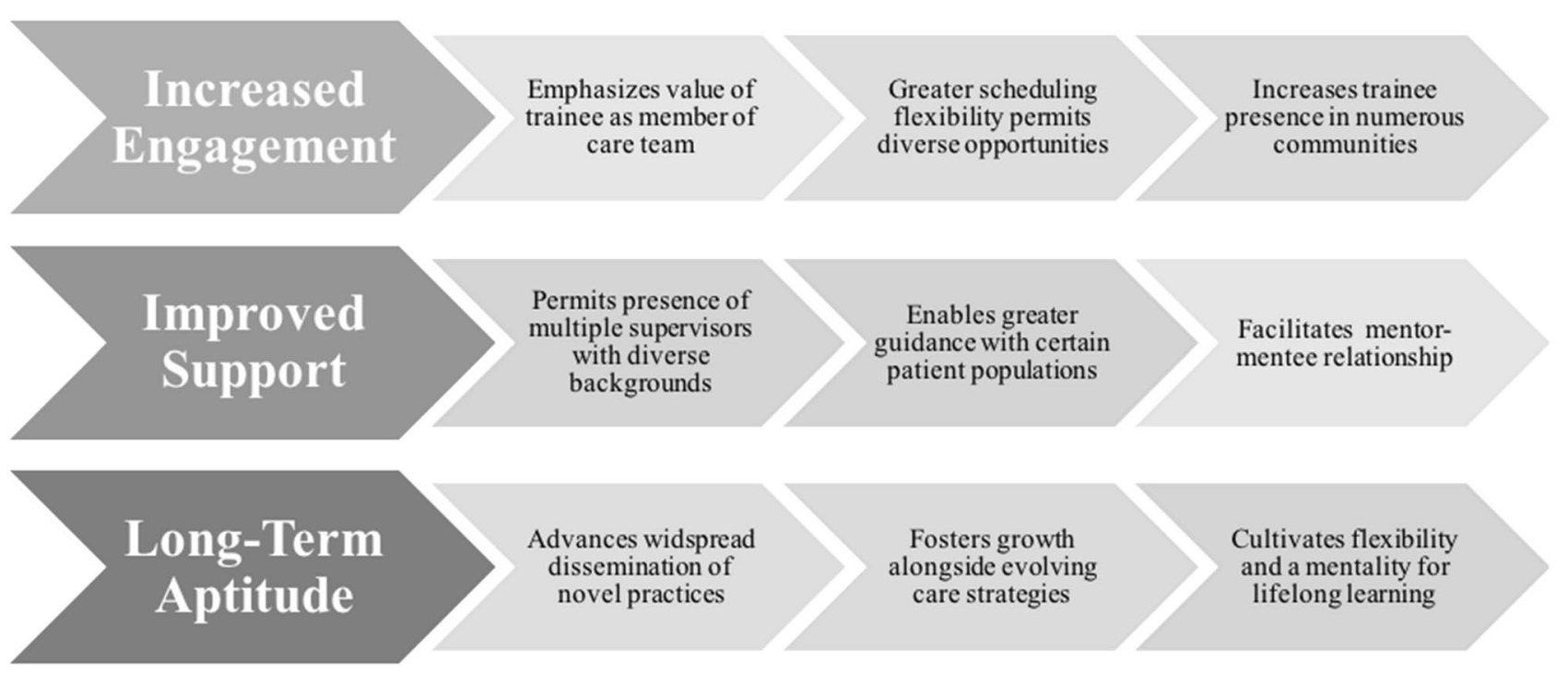

Fig. 3 Benefits of trainee involvement in the provision of virtual mental health care

to professional development, and learner involvement in institutional responses to COVID-19 were found to be an excellent arena for this to take place.

Regarding the other arm of the Coping Clinic, uptake of the Nurse Manager Consultation was strong as seen by a total of 28 hospital units requesting consultation. Given that this large number of requests could not be covered by health psychology learners alone, health psychology residents took on the role of consulting to the peer supporters at their bi-weekly debriefing meetings as more peer supporters became involved. Thus, psychology learners were consulting to providers either directly or indirectly on a regular basis since early in the pandemic.

\section{Discussion}

Determining how to deliver mental health care to a wide diversity of HCWs and learners required rapid mobilization of resources and personnel across a broad array of platforms. Academic medical centers are at risk of long-term effects related to the immediate and prolonged emotional response to stress from the COVID-19 pandemic across all levels of HCWs and learners. The impact of these stressors create immediate mental health needs, as well as long-term issues related to the prolonged nature of the event, changes in training and career trajectories, and modifications to home and work environments. MCW was able to utilize psychological first aid, cognitive behavioral and other psychological techniques, as well as the literature on disaster intervention, to create a platform of services intended to reach across the institution, affiliates, and HCWs within the community.
At the core of service mobilization were peer virtual support groups led by trained mental health practitioners that were designed to meet the needs of all types of health-care staff and faculty. This format provided the framework to create services to address the needs of learners, while also providing opportunities for learners to serve as providers at a time when many were removed from clinical experiences. Additionally, the action of pivoting existing student-led clinics to virtual platforms to provide immediate interventions for HCWs allowed expansion into advocacy and peer support throughout the institution. Further, the quick success of these formats allowed the creation of an additional virtual clinic that enabled psychological services to also extend to the health-care community surrounding the institution.

Our results can be understood within the context of previous guidelines and recommendations in the disaster literature associated with supporting HCWs exposed to trauma. MCW heavily relied upon the research literature to implementing evidence-based strategies (e.g., PFA, CBT) to mitigate distress and improve resilience in $\mathrm{HCWs}$ involved in disasters (Brooks et al., 2019; Surya et al., 2017).

Our results can also be understood within the existing research on support groups, which are often seen as a primary mode of providing support and enhancing emotional coping during challenging times (Davidson et al., 2012; Goldstrom et al., 2006). Many support groups occur in face-to-face settings, although many have transitioned to online formats over the past several years, as the needs of those seeking health care have diversified (Hai et al., 2021). Challenges of such groups have been well documented, from technological difficulties to a lack of recognition of the same non-verbal cues as in person (Banbury et al., 2018; Richardson et al., 2009). 
While research suggests that individuals who seek online care may have different personality styles than others, in a time of physical distancing when traditional care is unavailable, those differences may not play as significant a role. Of the research that has attempted to evaluate online support groups, methodologies have varied significantly, leading to a lack of universal best-practice guidance (White \& Dorman, 2001). Given that some research indicates that support groups tend to be most successful when facilitated by those with training (vs. lay peers) who can intervene and recognize emotional crises (Gilat et al., 2012), MCW utilized the approach of building support groups with trained facilitators.

The facilitators came from a variety of backgrounds and experiences, with many working in mental health. It is possible that certain knowledge, skills, or attributes may be key to effective group leadership (Pomery et al., 2016) and could be an area of future study to inform selection and training of facilitators. Further, while past research evaluated the effectiveness of support group interventions based on severity of illness symptoms or rarity of conditions, results also suggest mental health symptoms can be reduced when utilizing support group versus wait list controls (Griffiths et al., 2012; Haik et al., 2019; Huber et al., 2018).

Despite the overall success of these clinics, it is probable that many HCWs experiencing distress due to the pandemic did not become engaged in any of the aforementioned programs. This may have been due in part to: (1) a reluctance to utilize telehealth platforms, especially initially; (2) concern about having notes recorded in the electronic medical record; (3) existing stigma; and (4) logistical and time barriers to receiving mental health care (Galbraith et al., 2020). Additionally, given the nature of the health-care response to COVID-19, it is possible that many of the frontline workers were in "crisis" mode, focusing their attention on critical tasks they needed to do get through their shifts during a rapidly changing landscape (e.g., obtaining and donning the correct PPE, meeting training goals, continuing to care for patients, dealing with the home fallout of COVID-19 including financial struggles and childcare). Additionally, the COVID-19 pandemic was originally thought to be an acute crisis, but as the situation evolved, it was determined to be a longer-term response. As such, many HCWs may have foregone their mental health concerns early to mid-pandemic with the belief that this was an acute stressor to be endured.

Similarly, as our strategy for administering medical care has evolved with our current climate, our strategy for ensuring that learners' professional development has also evolved. We have learned that early exposure to different service formats and various care environments has been and will continue to remain crucial to help learners develop necessary aptitude and flexibility for future care. For these reasons, learner involvement should not only be encouraged, but necessitated and considered throughout the development of newly adapted virtual care programming. This will help to not only meet the goal of advancing learners' professional development but also to help minimize the lag between modality development and widespread dissemination of these new modalities.

From learning to adapt training models to ensure learners' professional development needs have been met to learning to provide virtual health care, the pandemic has ensured that we refocus upon remaining responsive in the face of future pandemics or other global events. Given this priority, next steps should focus upon evaluating the depth and breadth of mental health impact on HCWs and learners, what coping mechanisms are most helpful, and what services, whether formal or informal, proved beneficial. Hospital administration should be looking to researchers to inform them of what could help to bolster support for a population that has been overburdened by this pandemic.

There are several limitations noted with this study. First, though there were multiple affiliated clinical sites involved with and affected by these efforts, it remains a single-institution study and thus may not be generalizable across other institutions and geographical areas. Second, considering the rapid progression of the COVID-19 crisis and the need for a timely response, available literature to inform the design and implementation of effective pandemic-specific interventions was lacking when these described interventions were launched. Finally, given the clear need for rapid program implementation, the infrastructure necessary to systematically collect and analyze high-quality process and outcomes data for these interventions could not be established to provide robust information regarding efficacy.

Future research should evaluate utilization of services based on participation and retention, as well as effectiveness of the services based on qualitative and quantitative data to measure well-being and burn out of participants over time. Next steps must also include focus groups across the academic community to capture lessons learned from this pandemic and identify organizational factors that must be addressed to create a culture that de-stigmatizes mental health symptoms and promotes wellness for HCWs and learners during this pandemic and beyond.

Author Contributions All authors contributed to the study conception and design. Material preparation and analysis was completed by KK, $\mathrm{PhD}$ and $\mathrm{JA}, \mathrm{PhD}$. The first draft of the manuscript was written by all authors and all authors commented on previous versions of the manuscript. All authors read and approved the final manuscript. KK-Corresponding Author; Conceptualization. JA-Conceptualization; Project Administration. SL-Writing-original draft preparation; Writingreviewing and editing. KL-Writing-original draft preparation. DCWriting-original draft preparation; Writing-reviewing and editing. WHD-Writing-original draft preparation; Writing-reviewing and editing. TdRC - Resources. HA-Writing-original draft preparation; 
Writing-reviewing and editing. JRO-Writing-original draft preparation. DP-Writing-original draft preparation.

Funding Partial funding support was provided by the Greater Milwaukee Foundation. The clinical activities of the Coping Clinic were supported by the Medical College of Wisconsin Department of Psychiatry and Behavioral Medicine and supported by Froedtert Hospital infrastructure shortfall.

Data Availability Not applicable.

Code Availability Not applicable.

\section{Declarations}

Conflict of interest Kristin H. Kroll, Sadie Larsen, Kelsey Lamb, W. Hobart Davies, David Cipriano, Terri A. deRoon-Cassini, Himanshu Agrawa, Deepa Pawar, Julie Owen and Jennifer N. Apps declare they have no financial interests.

Ethical Approval Not applicable.

Consent to Participate MCW IRB PRO000037941 secured for collection of any surveys completed voluntarily in relation to Peer Support Group facilitation or participation.

Consent to Publish Not applicable.

Human and Animal Rights All procedures performed in studies involving human participants were in accordance with the ethical standards of the 1964 Helsinki declaration and its later amendments or comparable ethical standards.

Informed Consent Not applicable.

\section{References}

Banbury, A., Nancarrow, S., Dart, J., Gray, L., \& Parkinson, L. (2018). Telehealth interventions delivering home-based support group videoconferencing: Systematic review. Journal of Medical Internet research, 20, e25. https://doi.org/10.2196/jmir.8090

Behrman, S., Baruch, N., \& Stegen, G. (2020). Peer support for junior doctors: A positive outcome of the COVID-19 pandemic? Future Health Care Journal, 7, e64-e66.

Brooks, S. K., Rubin, G. J., \& Greenberg, N. (2019). Traumatic stress within disaster-exposed occupations: Overview of the literature and suggestions for the management of traumatic stress in the workplace. British Medical Bulletin, 129(1), 25-34.

Brown, G., Stanley, B., Western Interstate Commission for Higher Education (WICHE), and the Suicide Prevention Resource Center. (2009). Safety Planning Guide: A Quick Guide for Clinicians. Retrieved on March 24, 2020 from http://www.sprc.org/sites/defau 1t/files/SafetyPlanningGuide\%20Quick\%20Guide\%20for\%20Cli nicians.pdf

Cabarkapa, S., Nadjidai, S. E., Murgier, J., \& Ng, C. H. (2020). The psychological impact of COVID-19 and other viral epidemics on frontline health care workers and ways to address it: A rapid systematic review. Brain, Behavior, \& Immunity_Health, 8, 100144. https://doi.org/10.1016/j.bbih.2020.100144

Cao, W., Fang, Z., Hou, G., Han, M., Xu, X., Dong, J., \& Zheng, J. (2020). The psychological impact of the COVID-19 epidemic on college students in China. Psychiatry Research, 287, 112934.
Center for the Study of Traumatic Stress. (2021). Sustaining the psychological well-being of caregivers while caring for disaster victims. Retrieved on May 30, 2021 from https://www.cstsonline.org/ resources/resource-master-list/sustaining-caregiving-and-psych ological-well-being-while-caring-for-disaster-victims

Connors, C., Durkhanin, V., March, A., Parks, J. A., Norvell, M., \& Wu, A. W. (2019). Peer support for nurses as second victims: Resilience, burnout, and job satisfaction. Journal of Patient Safety and Risk Management, 25, 22-28.

Davidson, L., Bellamy, C., Guy, K., \& Miller, R. (2012). Peer support among persons with severe mental illnesses: A review of evidence and experience. World Psychiatry: Official Journal of the World Psychiatric Association (WPA), 11, 123-128. https:// doi.org/10.1016/j.wpsyc.2012.05.009

Epstein, R., \& Krasner, M. (2013). Physician resilience: What it means, why it matters, and how to promote it. Academic Medicine, 88, 301-303. https://doi.org/10.1097/ACM.0b013e3182 $80 \mathrm{cff} 0$

Galbraith, N., Boyda, D., McFeeters, D., \& Hassan, T. (2020). The mental health of doctors during the Covid-19 pandemic. BJPsych Bulletin. https://doi.org/10.1192/bjb.2020.44

Gilat, I., Tobin, Y., \& Shahar, G. (2012). Responses to suicidal messages in an online support group: Comparison between trained volunteers and lay individuals. Social Psychiatry and Psychiatric Epidemiology, 47, 1929-1935.

Greenberg, N., Docherty, M., Gnanapragasam, S., \& Wessely, S. (2020). Managing mental Health challenges faced by health care workers during covid-19 pandemic. BMJ, 368, m1211.

Goldstrom, I. D., Campbell, J., Rogers, J. A., Lambert, D. B., Blacklow, B., Henderson, M. J., \& Manderscheid, R. W. (2006). National estimates for mental health mutual support groups, self-help organizations, and consumer-operated services. Administration and Policy in Mental Health, 33, 92-103. https://doi.org/10.1007/s10488-005-0019-x

Griffiths, K., Mackinnon, A., Crisp, D., Christensen, H., Bennett, K., \& Farrer, L. (2012). The effectiveness of an online support group for members of the community with depression: A randomised controlled trial. PLOS One, 7(12), e53244. https://doi. org/10.1371/journal.pone.0053244

Hai, A. H., Lee, C. S., Oh, S., Vaughn, M. G., Piñeros-Leaño, M., Delva, J., \& Salas-Wright, C. P. (2021). Trends and correlates of Internet support group participation for mental health problems in the United States, 2004-2018. Journal of psychiatric research, 132, 136-143. https://doi-org.proxy.lib.mcw.edu. https://doi.org/10.1016/j.jpsychires.2020.10.012

Haik, D., Kashanchi, K., Tajran, S., Heilbronn, C., Anderson, C., Francis, D., Gelbard, A., \& Verma, S. (2019). The online support group as a community: A thematic content analysis of an online support group for idiopathic subglottic stenosis. The Annals of Otology, Rhinology, and Laryngology, 128, 293-299.

Huber, J., Muck, T., Maatz, P., Keck, B., Enders, P., Maatouk, I., \& Ihrig, A. (2018). Face-to face vs online peer support groups for prostate cancer: A cross-sectional comparison study. Journal of Cancer Survivors, 12, 1-9.

Institute of Medicine. (2012). Crisis standards of care: A systems framework for catastrophic disaster response: Volume 1: Introduction and CSC framework. The National Academies Press.

Inter-Agency Standing Committee Reference Group on Mental Health and Psychosocial Support in Emergency Settings. (2020, February). Interim briefing note addressing mental health and psychosocial aspects of COVID-19 outbreak. Inter-Agency Standing Committee. https://interagencystandingcommittee.org/ iasc-reference-group-mental-health-and-psychosocial-supportemergency-settings/interim-briefing

Lai, J., Ma, S., Wang, Y., Cai, Z., Hu, J., Wei, N., Wu, J., Du, H., Chen, T., Li, R., Tan, H., \& Hu, S. (2020). Factors associated 
with mental health outcomes among health care workers exposed to coronavirus disease 2019. JAMA Network Open, 3, e203976.

Lee, S. M., Kang, W. S., Cho, A. R., Kim, T., \& Park, J. K. (2018). Psychological impact of the 2015 MERS outbreak on hospital workers and quarantined hemodialysis patients. Comprehensive Psychiatry, 87, 123-127.

Louise Duncan, D. (2020). What the COVID-19 pandemic tells us about the need to develop resilience in the nursing workforce. Nursing Management (Harrow, London, England), 27, 22-27.

Maheu, M., Drude, K., Hertlein, K., Lipshutz, R., Wall, K., \& Hilty, D. (2019). Correction to: An interprofessional framework for telebehavioral health competencies. Journal of Technology in Behavioral Science. https://doi.org/10.1007/s41347-019-00113-x

Maunder, R., Hunter, J., Vincent, L., Bennett, J., Peladeau, N., Leszcz, M., Sadavoy, J., Verhaeghe, L. M., Steinberg, R., \& Mazzulli, T. (2003). The immediate psychological and occupational impact of the 2003 SARS outbreak in a teaching hospital. Cmaj, 168, $1245-1251$.

Magruder, K., Kassam-Adams, N., Thoresen, S., \& Olff, M. (2016). Prevention and public health approaches to trauma and traumatic stress: A rationale and a call to action. European Journal of Psychotraumatology. https://doi.org/10.3402/ejpt.v7.29715

Omer, S., Malani, P., \& del Rio, C. (2020). The COVID-19 pandemic in the US: A clinical update. JAMA, 323, 1767-1768. https://doi. org/10.1001/jama.2020.5788

Paladino, L., Sharpe, R. P., Galwankar, S. C., Sholevar, F., Marchionni, C., Papadimos, T. J., Paul, E., Hansoti, B., Firstenberg, M., Garg, M., Watson, M., \& (ACAIM), A. C. o. A. I. M. (2017). Reflections on the Ebola Public Health Emergency of International Concern, Part 2: The unseen epidemic of posttraumatic stress among healthcare personnel and survivors of the 2014-2016 Ebola outbreak. Journal of Global Infectious Diseases, 9, 45-50.

Pomery, A., Schofield, P., Xhilaga, M., \& Gough, K. (2016). Skills, knowledge and attributes of support group leaders: A systematic review. Patient Education and Counseling, 99, 672-688. https:// doi.org/10.1016/j.pec.2015.11.017

Posner, K., Brown, G., Stanley, B., Brent, D., Yershova, K., Oquendo, M., Currier, G., Melvin, G., Greenhill, L., Shen, S., \& Mann, J. (2011). The Columbia-Suicide Severity Rating Scale: Internal validity \& internal consistency findings from three multisite studies of adolescents and adults. American Journal of Psychiatry, 168, 1266-1277. https://doi.org/10.1176/appi.ajp.2011.10111704

Preti, E., Di Mattei, V., Perego, G., Ferrari, F., Mazzetti, M., Taranto, P., Di Pierro, R., Madeddu, F., \& Calati, R. (2020). The psychological impact of epidemic and pandemic outbreaks on health care workers: Rapid review of the evidence. Current Psychiatry Reports. https://doi.org/10.1007/s11920-020-01166-z

Richardson, L., Frueh, B., Grubaugh, A., Egede, L., \& Elhai, J. (2009). Current directions in videoconferencing tele-mental health research. Clinical Psychology: Science and Practice, 16, 323-338. https://doi.org/10.1111/j.1468-2850.2009.01170.x

Rojas, S., Carter, S., McGinn, M., \& Reger, M. (2019). A review of telemental health as a modality to deliver suicide-specific interventions for rural populations. Telemedicine \& e-Health. https:// doi.org/10.1089/tmj.2019.0083

Rotenstein, L. S., Ramos, M. A., Torre, M., Segal, J. B., Peluso, M. J., Guille, C., Sen, S., \& Mata, D. A. (2016). Prevalence of depression, depressive symptoms, and suicidal ideation among medical students: A systematic review and meta-analysis. JAMA, 316, 2214-2236.

Roycroft, M., Wilkes, D., Fleming, S., Pattani, S., \& Olsson-Brown, A. (2020). Preventing psychological injury during the covid-19 pandemic. BMJ, 369, m1702.

Salehi, M., Amanat, M., Mohammadi, M., Salmanian, M., Rezaei, N., Saghazadeh, A., \& Garakani, A. (2021). The prevalence of post-traumatic stress disorder related symptoms in Coronavirus outbreaks: A systematic-review and meta-analysis. Journal of Affective Disorders, 282, 527-538. https://doi.org/10.1016/j.jad. 2020.12.188

SAMHSA. (2015). Disaster technical assistance center supplemental research bulletin: Disaster behavioral interventions inventory. Retrievedo on May 28, 2021 from https://www.samhsa.gov/sites/ default/files/dtac/supplemental-research-bulletin-may-2015-disas ter-behavioralhealth-interventions.pdf

Seligman, M. (2011). Flourish. Free Press.

Shanafelt, T., Ripp, J., \& Trockel, M. (2020). Understanding and addressing sources of anxiety among health care professionals during the COVID-19 pandemic. JAMA, 323(21), 2133-2134. https://doi.org/10.1001/jama.2020.5893

Shen, X., Zou, X., Zhong, X., Yan, J., \& Li, L. (2020). Psychological stress of ICU nurses in the time of COVID-19. Critical Care, 24, 200. https://doi.org/10.1186/s13054-020-02926-2

Surya, M., Jaff, D., Stilwell, B., \& Schubert, J. (2017). The importance of mental well-being for health professionals during complex emergencies: It is time we take it seriously. Global Health: Science and Practice, 5, 188-196.

Tawfik, D., Profit, J., Morgenthaler, T., Satele, D., Sinsky, C., Dyrbye, L., Tutty, M., West, C., \& Shanafelt, T. (2018). Physician burnout, well-being, and work unit safety grades in relationship to reported medical errors. Mayo Clinic Proceedings, 93, 1571-1580. https:// doi.org/10.1016/j.mayocp.2018.05.014

Tugade, M. M., Shiota, M. N., \& Kirby, L. D. (Eds.). (2014). Handbook of positive emotions. Guildford Press.

Tracy, D. K., Tarn, M., Eldridge, R., Cooke, J., Calder, J. D. F., \& Greenberg, N. (2020). What should be done to support the mental health of health care staff treating COVID-19 patients? The British Journal of Psychiatry: the journal of mental science, 217(4), 537-539. https://doi.org/10.1192//bjp.2020.109

Vernberg, E., Steinberg, A., Jacobs, A., et al. (2008). Innovations in disaster mental health: PFA. Professional Psychology: Research and Practice, 39, 381-388. https://doi.org/10.1037/a0012663

White, M., \& Dorman, S. (2001). Receiving social support online: Implications for health education. Health Education Research, $16,693-707$.

World Health Organization. (2020, March 18). Mental Health and Psychosocial Considerations during the COVID-19 Outbreak. https://www.who.int/docs/default-source/coronaviruse/mentalhealth-considerations.pdf?sfvrsn=6d3578af_2

Wu, Y., Chen, C., \& Chan, Y. (2020). The outbreak of COVID-19: An overview. Journal of the Chinese Medical Association, 83, 217-220. https://doi.org/10.1097/JCMA.0000000000000270

Publisher's Note Springer Nature remains neutral with regard to jurisdictional claims in published maps and institutional affiliations. 